\title{
Transfusion-associated circulatory overload (TACO): prevention, management, and patient outcomes
}

\author{
Nareg H Roubinian ${ }^{1,2}$ \\ Edward L Murphy'-3 \\ 'Blood Systems Research Institute, \\ ${ }^{2}$ Department of Laboratory Medicine, \\ ${ }^{3}$ Department of Epidemiology and \\ Biostatistics, University of California \\ San Francisco, San Francisco, CA, \\ USA
}

This article was published in the following Dove Press journal:

International Journal of Clinical Transfusion Medicine

24 April 2015

Number of times this article has been viewed

\begin{abstract}
Transfusion-associated circulatory overload (TACO) is acute pulmonary edema associated with left atrial hypertension or volume overload occurring within 6 hours following a blood transfusion. Recognized by physicians as a common complication of blood transfusion, its incidence has been difficult to measure because active surveillance is required to counteract underreporting; active surveillance indicates overall incidence rates as high as $1 \%$ per transfused patient. Recent clinical and translational research has focused on the development of electronic alert systems to measure TACO incidence and provide alerts to physicians regarding patients at high risk. Translational research regarding the utility of biomarkers such as brain natriuretic protein (BNP) or N-terminal pro-BNP (NT-proBNP) as diagnostic tools for TACO has been only moderately successful, but the search for other biomarkers continues. Prevention strategies can be developed based upon evidence derived from both observational studies and might include: using documented risk factors to highlight patients at risk, preferably using real-time analysis of electronic medical records; implementation of modified transfusion strategies to minimize the volume and infusion rate of blood products; consideration of prophylactic diuretic therapy; and heightened diagnostic awareness combined with rapid implementation of treatment. Randomized clinical trials will be required to test such strategies before they are widely implemented. Finally, the occurrence of TACO ought to be considered as a potentially avoidable medical complication that could be used to benchmark transfusion and critical care practice across hospitals.
\end{abstract}

Keywords: blood transfusion, pulmonary edema, risk factors, prevention and control, therapeutics

\section{Introduction}

Pulmonary complications of transfusion include transfusion related acute lung injury (TRALI), transfusion-associated circulatory overload (TACO), and other types of acute lung injury (ALI). TACO, likely the most common of these, is defined as symptoms and signs of acute pulmonary edema associated with left atrial hypertension or volume overload occurring within 6 hours of blood transfusion. These signs include acute respiratory distress, hypertension, hypoxemia, findings of congestive heart failure including elevated and an S3 heart sound as well as vascular resdistribution, Kerley B line, and bilateral opacities on chest X-ray. ${ }^{1}$ The term TACO was coined by Popovsky following an early article on posttransfusion pulmonary edema and reviewed 5 years ago by Popovsky in his Emily Cooley lecture in 2009. ${ }^{2,3}$ The differential diagnosis between TACO and TRALI can be difficult, the distinction being primarily whether hydrostatic versus capillary leak syndrome is responsible for the pulmonary edema. ${ }^{1,4,5}$

Correspondence: Edward L Murphy Blood Systems Research Institute, 270 Masonic Avenue, San Francisco, CA 94II8, USA

Tel + I 4157496668

Fax + I 4I5 90I 0733

Email ed.murphy@ucsf.edu
International Journal of Clinical Transfusion Medicine 20।5:3 17-28 
The Centers for Disease Control and Prevention (CDC) has proposed criteria for the diagnosis of TACO as part of the its hemovigilance network, including a minimum of three signs of pulmonary edema within 6 hours of blood transfusion in the absence of other causes of ALI. ${ }^{6}$

Beyond the dogma that TACO is caused by the infusion of too much blood too quickly, risk factors for TACO are not well understood. Infusion rate may be more important than the total volume of blood transfused, however the optimal infusion rate of blood components and adjustment for patient characteristics are poorly defined. ${ }^{7,8}$ As described below, host factors are probably more important than infusion practice. Patients older than 60 years and women predominate in several case series. ${ }^{9-13}$ A positive fluid balance and chronic renal failure have also been implicated. The role of underlying cardiac disease, although implicitly recognized, has not been well described. Understanding these and other host factors could be used to construct a predictive algorithm useful to clinicians, as has been done recently for the prediction of sudden cardiac death in patients with coronary artery disease. ${ }^{14}$

TACO continues to represent a significant cause of posttransfusion morbidity and mortality, but several authors have commented on the relative scarcity of published research on this important and common clinical problem. In particular, good quantitative studies of patient risk factors and clinical practices that are associated with TACO are lacking. If patient risk factors could be better defined, algorithms could be devised to indicate which patients are at highest risk, and transfusion rates could be reduced or prophylactic diuretics administered in these patients. A better understanding of the evidence supporting the prevention and treatment of TACO also fits well with current emphases on hemovigilance as well as efforts to identify and minimize iatrogenic complications and errors in medical practice that contribute significantly to the costs of healthcare.

In this review, we shall therefore first touch upon the current literature regarding the pathophysiology and risk factors for TACO, including patient-specific, practice-spectific, and biomarkers. We shall then review what is known about the diagnosis and treatment of TACO, focusing on oxygenation, fluid balance, underlying cardiac function, and differential diagnosis from other pulmonary transfusion reactions. Potential preventative measures will be considered along with evidence supporting their efficacy, and information on adverse outcomes associated with TACO will be reviewed. We shall finish with an assessment of gaps in the evidence base and suggestions for future research. We have limited this review to the adult as opposed to pediatric literature.

\section{Pathophysiology of TACO}

The pathophysiology of TACO resembles that of other types of acute hydrostatic pulmonary edema. ${ }^{15,16}$ In a patient without pulmonary edema, pulmonary capillary pressure is counterbalanced by colloid osmotic pressure within the capillaries; small amounts of transudate are removed from the alveoli by the pulmonary lymphatic system (Figure 1). With normal cardiovascular function, there is a range of left atrial and pulmonary capillary pressures during which homeostasis is maintained by lymphatic drainage; beyond a critical "tipping point", however, there is a linear relationship between increasing left atrial pressures and the rate of pulmonary edema formation. ${ }^{16}$ Pulmonary edema occurs in patients with heart failure without elevations of total blood volume. In TACO, a moderate increase in blood volume and pulmonary capillary pressure mediated by the blood transfusion pushes patients with compensated cardiac dysfunction or chronic renal failure beyond the tipping point into pulmonary edema. In patients with normal hearts and kidneys, massive transfusion is typically required to tip them into hydrostatic pulmonary edema.

\section{Incidence of TACO and associated risk factors and biologic markers}

Although TACO represents the second most common cause of transfusion related deaths reported to the Food and Drug Administration in fiscal year 2012, ${ }^{17}$ the incidence of TACO is poorly defined. ${ }^{18,19}$ In retrospective or prospective cohorts with some degree of active surveillance, it ranges from $1 \%$ to $8 \%$ of patients transfused (Table 1). ${ }^{9,20,21}$ Hemovigilance data tend to give much lower incidence rates, probably due to underreporting of a complication which may be viewed as a problem in transfusion administration rather than a defect in the blood product. ${ }^{22,24}$ There is also underestimation of the severity of morbidity and mortality associated with TACO. ${ }^{7}$

Risk factors for and markers of TACO can be divided into those that are related to the patient, including demographics and coexisting disease; those that are related to clinical practice, including the volume and infusion rate of blood products other fluids and overall fluid balance management; and markers of underlying pathophysiology, including brain natriuretic protein (BNP) or N-terminal pro-BNP (NT-proBNP) and other biomarkers (Table 2).

Patient-specific risk factors clearly include age over 60 or 70 years. ${ }^{9-13}$ Age is likely a surrogate for other predisposing risk factors such as preexisting cardiac failure or chronic kidney disease. In the pediatric literature (not covered 


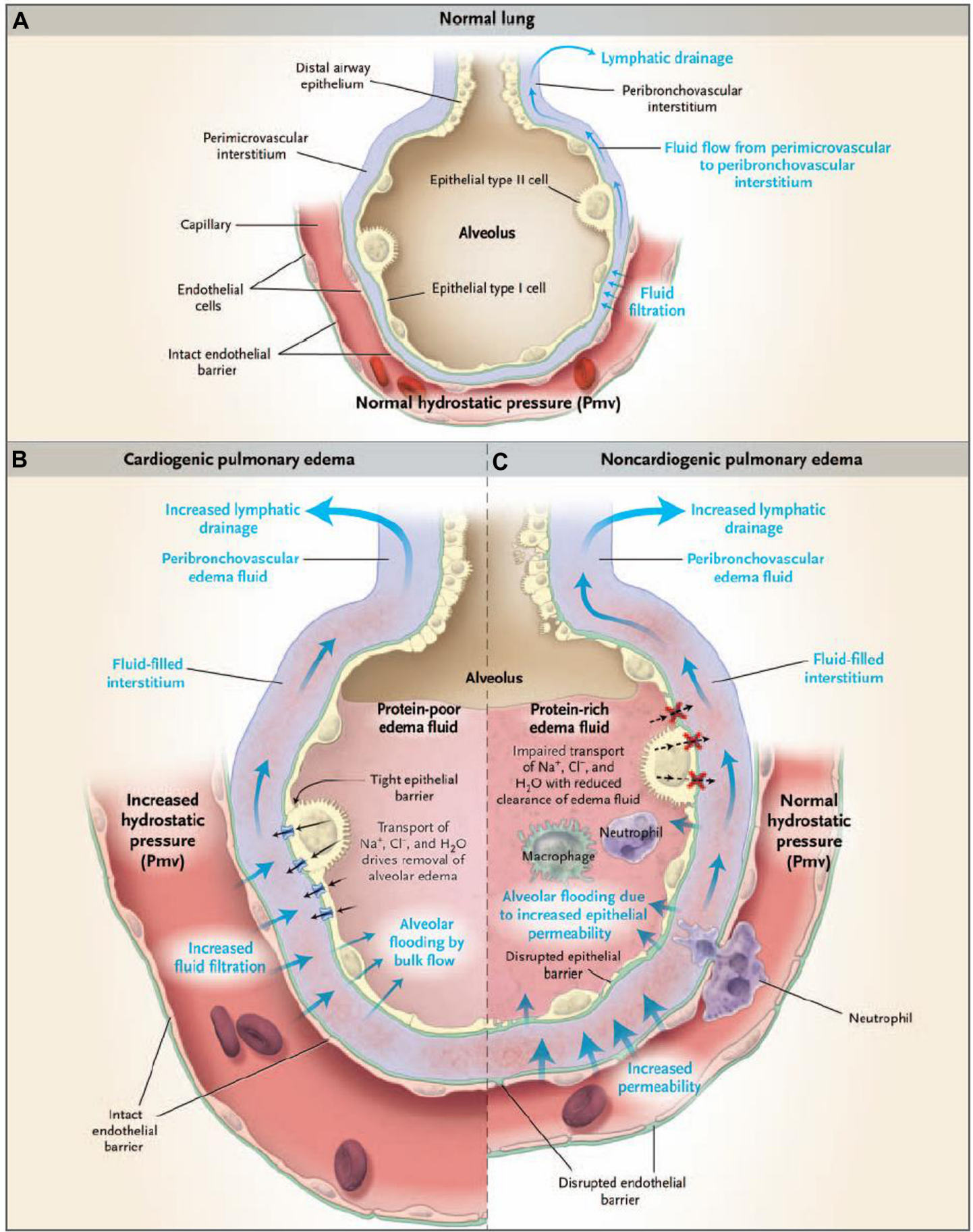

Figure I Physiology of Microvascular Fluid Exchange in the Lung.

Notes: In the normal lung (A), fluid moves continuously outward from the vascular to the interstitial space according to the net difference between hydrostatic and protein osmotic pressures, as well as to the permeability of the capillary membrane. When hydrostatic pressure increases in the microcirculation such as in TACO, the rate of transvascular fluid filtration rises (B). Since the permeability of the capillary endothelium remains normal, the filtered edema fluid leaving the circulation has a low protein content. Noncardiogenic pulmonary edema $(\mathbf{C})$ occurs when the permeability of the microvascular membrane increases because of direct or indirect lung injury (including transfusion related acute lung injury), resulting in a marked increase in the amount of fluid and protein leaving the vascular space. From The New England Journal of Medicine, Ware LB, Matthay MA, Clinical practice. Acute pulmonary edema. 353(26):2788-2796. Copyright @ (2005) Massachusetts Medical Society. Reprinted with permission from Massachusetts Medical Society. ${ }^{15}$ Abbreviation: TACO, transfusion-associated circulatory overload.

here), some authors also mention very young age (less than 3 years) as a risk factor. Female sex is another clear risk factor which may be confounded with smaller body size for which an equivalent transfusion volume represents a greater hemodynamic load. ${ }^{9-13}$ However it is intriguing to speculate whether sex specific differences could be related to differences in the prevalence of underlying diastolic heart failure cytokines or sex-specific differences in underlying physiology 
Table I Incidence of TACO by type of surveillance used to find cases

\begin{tabular}{|c|c|c|}
\hline Authors and year & Patient population & Incidence \\
\hline \multicolumn{3}{|l|}{ Passive surveillance } \\
\hline Kleinman et al $2003^{22}$ & Quebec hemovigilance (RBC) & $0.042 \%$ \\
\hline $\begin{array}{l}\text { AFSSAPS Annual } \\
\text { report } 2009^{23}\end{array}$ & French hemovigilance & $0.056 \%^{\mathrm{a}}$ \\
\hline Bolton-Maggs $2014^{24}$ & British SHOT hemovigilance & $\leq 0.01 \%{ }^{\mathrm{b}}$ \\
\hline \multicolumn{3}{|l|}{ Active surveillance } \\
\hline Popovsky et al $1996^{20}$ & 382 Orthopedic patients & $1.05 \%$ \\
\hline Bierbaum et al $1999^{21}$ & I499 Orthopedic patients & $8.01 \%$ \\
\hline Rana et al $2006^{9}$ & 2297 ICU patients & $1.09 \%$ \\
\hline Narick et al $2012^{79}$ & 84 plasma recipients & $4.8 \%$ \\
\hline
\end{tabular}

Notes: aln patients aged 55 years and older; 'SHOT does not provide a denominator of transfused patients. During 2013, they reported 96 cases in a system that transfused 2.04 million RBC. Assuming an average of two RBC units per transfusion episode, TACO incidence was $\leq 0.01 \%$.

Abbreviations: TACO, transfusion-associated circulatory overload; RBC, red blood cell; SHOT, serious hazards of transfusion; ICU, intensive care unit.

as indicated by biomarkers. Other clear risk factors include preexisting cardiac dysfunction, which makes sense since diminished cardiac reserve would be less likely to handle an additional volume load, ${ }^{11,12,25,26}$ and chronic renal failure which would make fluid homeostasis more difficult. ${ }^{11,12,25}$ Patient-specific risk factors mentioned by a minority of studies include white race/ethnicity, ${ }^{12}$ hemodynamic shock prior to the onset of TACO, ${ }^{25}$ a history of various types of anemia, ${ }^{12}$

Table 2 Risk factors for TACO

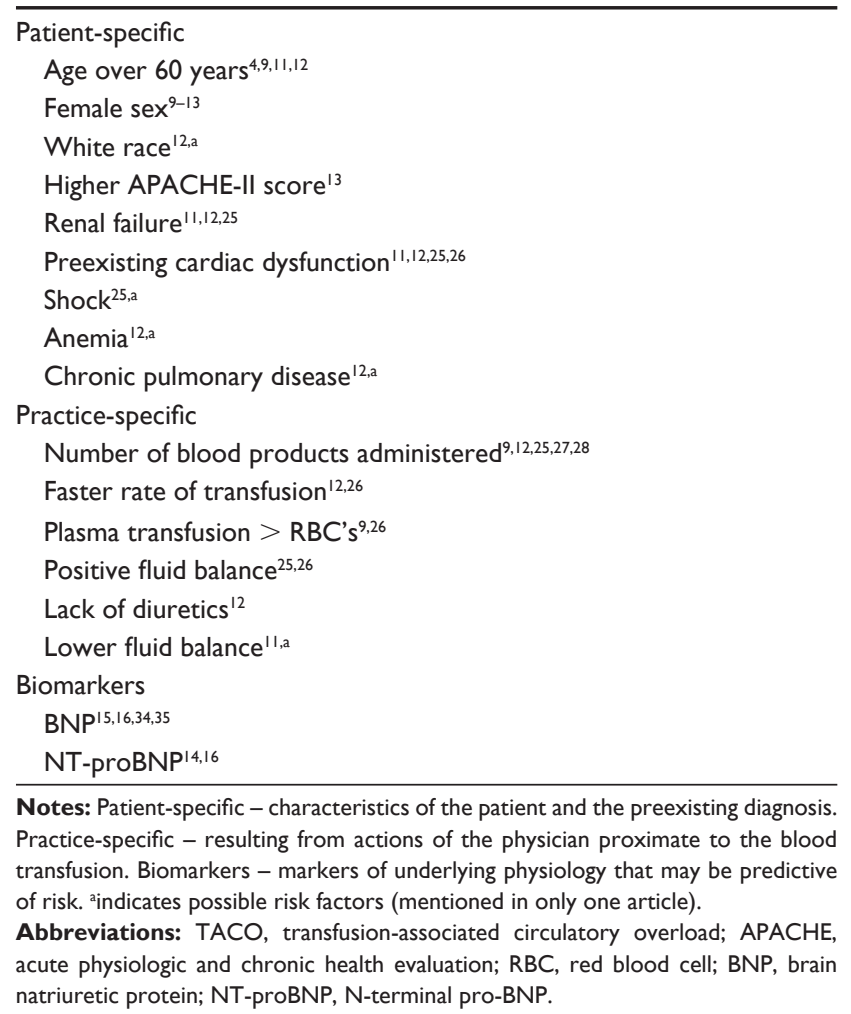

and a history of chronic pulmonary disease. ${ }^{12}$ These must be considered potential risk factors as biologic plausibility is lacking (race/ethnicity), or the risk factor is associated with a need for increased transfusion (shock, anemia). Practicespecific risk factors include the total volume of blood products administered, with consistent findings regardless of red blood cell, platelet, or plasma transfusion. ${ }^{9,12,25,27,28}$ There is some indication that plasma transfusion may be associated with an increased risk of TACO compared to other blood products, but this may be confounded by the total volume of transfusion administered. A faster blood product infusion rate was implicated by two studies and is a plausible risk factor; however this granular data is often not available which may explain why its role is not better understood..$^{12,26}$ A positive fluid balance preceding transfusion also makes pathophysiologic sense as the additional volume challenge posed by transfusion is less likely to be tolerated by patients who have received significant intravenous fluids at the start of transfusion. ${ }^{25,26}$ Interestingly, several studies found a similar or lower fluid balance in TACO as compared to TRALI suggesting that this variable may not be as useful to differentiate these syndromes. ${ }^{11,13,29,35}$

Rapid evaluation of biomarkers has had a major impact in clinical diagnosis and decision making. The use of BNP in classification of cardiogenic pulmonary edema spawned interest in its ability to identify patients at risk for TACO. A TACO case-control evaluation showed elevated BNP levels in patients with TACO in comparison to levels in patients who did not develop pulmonary edema following transfusion, and other retrospective analyses have found similar elevations in patients with TACO. ${ }^{10,30,31}$

The role of BNP in the differential diagnosis of transfusion-related lung edema is less clear. Studies have found overall higher BNP and NT-proBNP levels in TACO patients in comparison to those in patients with TRALI and Possible TRALI, defined as a TRALI syndrome when other risk factors for pulmonary edema such as sepsis or pneumonia are present. ${ }^{11,31,32}$ However, BNP levels alone were found to have limited ability in differentiating these groups of patients from one another. Another caveat to the above studies is that they did not fully control for the change in specificity of BNP in patients with acute and chronic renal insufficiency. This factor, in addition to the need for a larger sample size, may explain the lack of diagnostic cut points for working definitions such as the CDC Biovigilance criteria, though others have postulated thresholds for TACO and TRALI. ${ }^{33}$ A practical challenge to better establishing cut points for $\mathrm{BNP}$ is recognition that it is not routinely used clinically in 
identifying transfusion-related complications. One review of TACO found that BNP values were only available in $3 \%$ (3/98) and $11 \%(11 / 98)$ of cases prior to and following the development of pulmonary edema, respectively. ${ }^{12}$

More recently, pretransfusion elevations in inflammatory cytokines have been recognized in patients who develop TRALI, and not TACO ${ }^{32,34-36} \mathrm{~A}$ recent study by the authors of this review found utility of these cytokines in differentiating cardiogenic and noncardiogenic pulmonary edema prior to and following transfusion. ${ }^{32}$ In recent years, a number of novel biomarkers (eg, Cystatin C, GDF-15, ST-2) have identified patients at higher risk of adverse cardiac and renal outcomes. ${ }^{37-39}$ Future studies are needed to examine the utility of these markers alone and in combination to risk stratify and differentiate patients with pulmonary transfusion reactions.

\section{Diagnosis of TACO: current evidence} Signs and symptoms of TACO typically begin within 6 hours of completion of blood transfusion and are typified by tachycardia, tachypnea, and often, hypertension. ${ }^{20,40,41}$ However, these findings may initially be subtle, especially in postoperative patients who are often already receiving supplemental oxygen. An elevated pulse pressure is sometimes recognized in patients with cardiogenic pulmonary edema, and recent evidence suggests that this finding may be accentuated in TACO patients relative to blood pressure changes seen in nontransfused patients. ${ }^{42}$ The symptoms of TACO are typically accompanied by the development of hypoxemia, and chest radiography is most often used to identify pulmonary edema as the cause amongst the many possible etiologies in hospitalized patients. ${ }^{15}$ Chest radiography, in addition to identifying pulmonary edema, may provide further evidence to support a cardiogenic etiology with findings of cardiomegaly and pleural effusions. While clearly a useful tool in the evaluation of TACO, a review of 100 TACO patients found that posttransfusion chest $\mathrm{X}$-ray was not universally ordered, occurring in $80 \%$ of patients. ${ }^{12}$ Whether a diagnosis of cardiogenic pulmonary edema was made on clinical signs and symptoms alone or if pulmonary edema was identified on pretransfusion chest $\mathrm{X}$-rays is not clear.

Hypoxia related to pulmonary edema is most often recognized through the use of pulse oximetry but may be missed in operative cases where supplemental oxygen is routinely given during and following surgery. In these cases, hypoxemia may be identified via measurements of arterial blood gases. ${ }^{43}$ Arterial blood gas testing is a standard tool used to assess patients in acute respiratory distress of unclear etiology. Measurement of the ratio of the partial pressure of oxygen to fractional inspired oxygen $\left(\mathrm{PaO}_{2} / \mathrm{FiO}_{2}\right)$ is a routine measure of pulmonary impairment used in the definition of TRALI and acute respiratory distress syndrome (ARDS). This measure allows one to quantify the degree of hypoxemia (eg, $\mathrm{PaO}_{2} / \mathrm{FiO}_{2}$ ratio $<300$ for diagnosis of TRALI) relative to administered oxygen, though caveats are often present in patients not receiving positive pressure ventilation. ${ }^{44}$ Similar thresholds for hypoxemia have not yet been defined for TACO and may be more difficult to delineate given its sometimes mild presentation and infrequent utilization of blood gases in one review. ${ }^{27}$ Nevertheless, arterial blood gas measurement provides similar insights into the severity of pulmonary compromise of TACO and TRALI.

Invasive hemodynamic monitoring may provide important information regarding a patient's volume status, especially in complex critically ill patients where the pathophysiology of a patient's pulmonary edema is often difficult to assess. ${ }^{45}$ Pulmonary capillary and central venous pressure monitoring requires placement of an invasive catheter in the appropriate vascular space for their assessment and experience in interpreting waveforms correctly. At least one large randomized controlled trial in patients with ARDS has not shown evidence for a benefit of pulmonary artery catheters in improving clinical outcomes. ${ }^{46}$ As a result, their use has decreased and subsequent retrospective reviews of TACO have found infrequent utilization of this form of cardiac monitoring. ${ }^{26}$

Echocardiography, prior to or following transfusion, provides useful noninvasive information regarding the pathogenesis of posttransfusion pulmonary edema. ${ }^{33,45}$ Quite often elderly hospitalized patients do not have a known history of congestive heart failure, and this may only be identified at the time of surgery or in the evaluation of posttransfusion pulmonary edema. ${ }^{12,33,45}$ Echocardiographic findings of systolic heart failure, such as left ventricular dilatation and reduced ejection fraction, are well defined, though findings of diastolic dysfunction or valvular abnormalities may not be readily identified due to arrhythmia or technical quality of imaging. ${ }^{47}$ Findings of left atrial enlargement often denote chronically elevated left sided filling pressures suggestive of pressure or volume overload. ${ }^{4-50}$ Likewise, the lack of respiratory variation of the inferior vena cava in spontaneously breathing patients is a measure of a patient's volume status analogous to invasive central venous pressure monitoring. ${ }^{51}$ Findings of systolic or diastolic dysfunction as well as elevated right or left sided filling pressures should add weight to a cardiogenic etiology when differentiating the etiologies of posttransfusion pulmonary edema. ${ }^{26,33,45}$ 
Clinical, echocardiographic, and laboratory markers are most helpful in differentiating cardiogenic from noncardiogenic pulmonary edema. ${ }^{52,53}$ These findings are most critical in differentiating cases of TACO from TRALI, while cases of Possible TRALI may have a mixture of permeability and hydrostatic pulmonary edema. For example, several studies have demonstrated elevated central venous pressures as well as laboratory markers of ventricular strain in cases of Possible TRALI, so these may not be useful in distinguishing this pulmonary syndrome from TACO. ${ }^{13,26}$

\section{Management of TACO}

Management of patients who develop TACO builds upon the well-described management of cardiogenic pulmonary edema. ${ }^{54}$ Some tenets of initial management are inherent such as providing supplemental oxygen, elevation of the head of the bed, and prescription of diuretics and nitrates as hemodynamics allow. ${ }^{15,55}$ Monitoring includes sequential evaluation of vital signs including continuous pulse oximetry and cardiac telemetry as well as strict measurement of urine output following administration of diuretics. Further administration of blood components and other intravenous fluids should be discontinued or limited as possible. ${ }^{56}$

For more severe cases at risk for respiratory failure, there should be consideration for transfer to a hospital setting where invasive or noninvasive positive pressure ventilation can be administered. Noninvasive ventilation has been shown to decrease the need for intubation, improve respiratory parameters, and possibly decrease mortality in patients with cardiogenic pulmonary edema. ${ }^{57-59}$ The use of nitrates and other vasodilators to reduce preload should be considered, especially in patients with significant hypertension, and renal replacement therapy may be necessary in patients with severe renal impairment. ${ }^{54,60}$

In these severe cases, distinguishing the etiology of posttransfusion pulmonary edema can be critical with distinct differences in respiratory and diuretic management. For example, in cases of TACO, aggressive diuretic therapy and noninvasive ventilation would be prescribed as the patient's cardiopulmonary status allows. However, utilization of a low tidal volume strategy via invasive mechanical ventilation and maintenance of normovolemia would be appropriate in patients with TRALI or Possible TRALI.

\section{Measures to prevent TACO and evidence for efficacy of same}

Measures to prevent TACO begin with accurate identification of patients at risk utilizing some form of active surveillance.
With the rapid expansion of electronic medical records, algorithms to screen and identify cases of posttransfusion pulmonary edema are becoming more common (Figure 2). ${ }^{13}$ The development of automated screening for high risk patients with comorbid medical conditions prior to transfusion is feasible using electronic medical record systems. ${ }^{61}$ One study utilized a simple TACO risk score that included age, findings suggestive of congestive heart failure, and renal dysfunction found that patients with higher scores received a smaller volume of transfused product before developing TACO compared with patients with a lower score. However, in this observational study, the use of diuretics did not correlate with increasing TACO risk scores. ${ }^{12}$ Clinical decision support systems similar to those under development for other medical complications and incorporating relevant vital signs such as pulse pressure or a patient's creatinine clearance could further alert clinicians to patients at high risk of developing TACO.$^{62}$ Additional pretransfusion cardiopulmonary biomarkers may be also relevant in identifying patients with comorbid risk factors more likely to develop TACO. ${ }^{11}$

Preventing TACO requires identification and understanding of the modifiable practice-based risk factors (Table 3). Several studies have found the overall number of units transfused as well as the rate of transfusion to be risk factors for the development of TACO. ${ }^{25,26,63}$ Evidence-based transfusion practice, reduction of transfusion infusion rates, and prophylactic volume reduction with diuretics may all limit the incidence of TACO. Findings from randomized clinical trials suggest that more liberal transfusion practice is associated

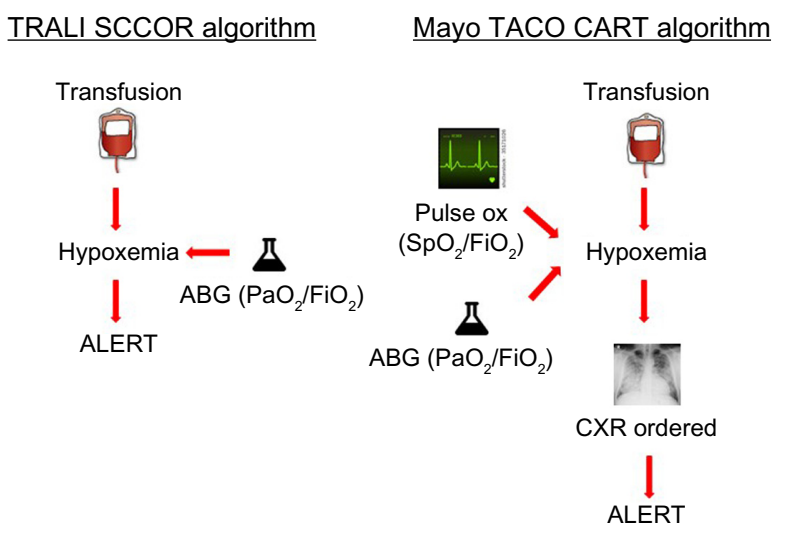

Figure 2 Comparison of automated screening algorithms for the detection of TACO and other pulmonary transfusion reactions.

Notes: The pioneering algorithm on the left relied upon linking blood product issuance with arterial blood gas results. ${ }^{82}$ When feasible, the more elaborated algorithm on the right offers potentially better sensitivity (due to the inclusion of pulse oximetry data to detect milder cases) and specificity (due to inclusion of a clinician's order for a chest radiograph). ${ }^{13}$

Abbreviations: TACO, transfusion-associated circulatory overload; SCCOR, Specialized Centers of Clinically Oriented Research; CART, classification and regression tree. 
Table 3 Prevention and therapy of TACO

\begin{tabular}{l} 
Primary prevention \\
Evidence-based transfusion practice \\
Single unit transfusion \\
Blood component alternatives \\
Secondary Prevention \\
Algorithms to identify high risk patients \\
Slower infusion rates in high risk patients \\
Prophylactic diuretics \\
Treatment of TACO \\
Oxygen supplementation \\
Cessation of transfusion \\
Diuretic therapy \\
Vasodilators \\
Positive pressure ventilation as needed \\
\hline Abbreviation: TACO, transfusion-associated circulatory overload.
\end{tabular}

with more frequent posttransfusion pulmonary edema in comparison to restrictive. ${ }^{27,28,64}$ Patient blood management strategies derived from these findings suggest transfusion of a single red blood cell unit at a time, and using a more restrictive hemoglobin threshold may reduce the likelihood of TACO. However, TACO has been associated with transfusion of a single red blood cell unit ${ }^{65}$ and Lieberman et al ${ }^{12}$ found that the mean pretransfusion hemoglobin level was $7.2 \mathrm{~g} / \mathrm{dL}$ in cases of TACO, in line with more restrictive practice. A nested case-control study with matching for risk factors showed that plasma administration for the reversal of anticoagulation was a risk factor for TACO.$^{26}$ These patients receiving often large volumes of plasma and red blood cells are commonly elderly individuals with impaired cardiac and renal function. The use of factor concentrates rather than plasma for this indication has been associated with a lower incidence of pulmonary edema in a small randomized controlled trial. ${ }^{66}$

There is currently no prospective data on the impact of lower blood transfusion infusion rates on the incidence of TACO. The rapid infusion of multiple blood components and medications plays a significant factor in the development of TACO, even when the overall fluid balance is not significantly different from that of TRALI or Possible TRALI. 26,33,35 In critically ill patients, blood component administration does not typically occur in isolation; other therapeutic agents such as vasopressors or antibiotics are often being infused as well. This is an all too common scenario in surgical patients when aggressive therapeutics necessary to maintain hemodynamics and control hemorrhage are balanced with the increased risk of TACO in patients with impaired cardiorenal function. While appropriate in patients with hemorrhagic shock, rapid administration of platelets and plasma has historically been utilized, especially in the case of the latter blood component where large volumes are often required to reverse coagulopathy. The efficacy of limiting infusion rates of blood components as well as other crystalloid fluids on the incidence of TACO remains unknown but is a reasonable practice even without evidence.

Diuretics may be appropriate in hemodynamically stable patients prior to or during blood transfusion and are often given as treatment after the development of pulmonary edema. The administration of prophylactic diuretics at the time of transfusion should be considered in hemodynamically stable patients with impaired cardiac function as well as in individuals with impaired but stable renal function. Supporting this practice, a study showed that the administration of diuretics resulted in unchanged cardiac filling pressures following transfusion, ${ }^{67}$ however prospective studies are needed to evaluate the benefit in preventing TACO. In practice, the use of diuretics as a treatment for TACO was found to be relatively infrequent (29\% and 27\%) in two studies. ${ }^{12,31}$ Whether this low level of utilization was due to the mild nature of the TACO event or concurrent hemodynamic instability is not clear. In a matched case-control study of risk factors of TACO ${ }^{26}$ pretransfusion diuretic was not found to be protective but also did not occur with high frequency. Therefore, further clinical research on the benefit of prophylactic diuretic therapy in preventing TACO appears to be needed.

Another area of translational research that may have a future impact upon TACO prevention concerns the preparation and storage of blood components. The hypothesis that inflammatory mediators play a role in the pathogenesis of TACO is supported by declines in reported incidence of TACO in addition to TRALI following universal leukoreduction. ${ }^{41,68} \mathrm{~A}$ randomized controlled trial examining the impact of age of red blood cells did not find differences in measures of pulmonary function, immunologic, or coagulation changes in fresh versus standard issue blood. ${ }^{69}$ Current research focuses on the impact of washing techniques and irradiation of red blood cells on markers of hemolysis and inflammation as well as the incidence of TACO. ${ }^{70-72}$

\section{Outcomes following TACO}

Despite the prevailing image of transient volume overload which can be treated easily and carries little morbidity, TACO is, in fact, associated with increased morbidity and probably increased mortality. Several studies have found that length of stay in the intensive care unit (ICU) and in the hospital is increased following an episode of TACO. Murphy et $\mathrm{al}^{25}$ (Figure 3) found that TACO was associated with increased length of stay in the ICU (Hazard Ratio [HR] for discharge, $0.37 ; 95 \%$ CI 

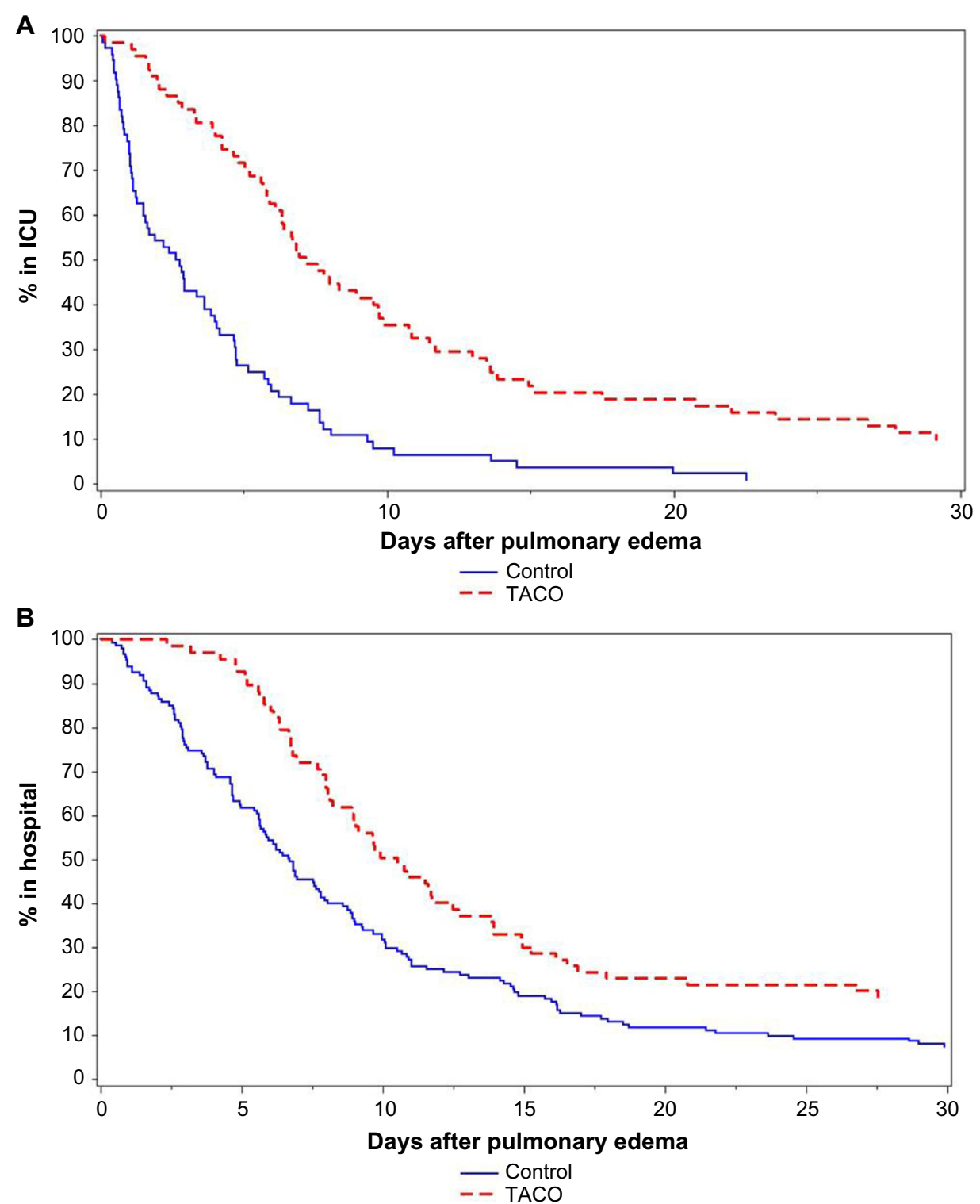

Figure 3 Length of stay in the (A) ICU and (B) hospital, in days, for TACO cases (dotted line) and transfused controls without pulmonary edema (solid line). Notes: These analyses counted length of stay from the time of the development of pulmonary edema (TACO) or 6 hours after transfusion (controls) until discharge from the ICU and hospital, respectively. Reprinted from The American Journal of Medicine, I26(4), Murphy EL, Kwaan N, Looney MR, et al, Risk factors and outcomes in transfusionassociated circulatory overload, e29-e38., Copyright (2013), with permission from Elsevier. ${ }^{25}$

Abbreviations: ICU, intensive care unit; TACO, transfusion-associated circulatory overload.

0.26-0.53) and in the hospital (HR for discharge, 0.64; 95\% CI $0.48-0.86)$. Li et $\mathrm{al}^{73}$ also found increased length of stay in both the ICU (3.0 versus 1.7 days) and hospital (9.4 versus 6.1 days; both $P<0.05)$. Menis et $\mathrm{al}^{74}$ also found in increased length of hospital stay ( $\mathrm{OR}=1.30$ for a stay of $7-10$ days). Magee and Zbrozek, ${ }^{75}$ in a multivariate analysis of discharge data from more than 600 United States hospitals, found an increase in ICU length of stay from 5.2 to 6.0 days $(P<0.001)$, hospital length of stay from 10.0 to 12.9 days $(P<0.001)$ and total hospital cost from $\$ 32,582$ to $\$ 46,644(P<0.001)$ for cases of fluid overload in association with plasma transfusion.
TACO may also increase mortality, although evidence for this is less consistent. Murphy et $\mathrm{al}^{25}$ found increased inhospital mortality following an episode of TACO, as well as prolonged ICU and hospital length of stay after pulmonary edema for patients who developed TACO compared with controls. Li et $\mathrm{al}^{73}$ found increased 5-year mortality following both TRALI and TACO, although the increased death rate in TACO cases was not statistically different from that of non-TACO cases $(P=0.144)$. Another study with a very small number of cases also suggested that patients with TACO had increased mortality and morbidity. ${ }^{20}$ Hebert et a $2^{27}$ 
found similar survival but higher risk of pulmonary edema in patients transfused with a liberal versus restrictive transfusion strategy.

Thus, it is reasonable to consider TACO as a potential killer and a cause of significant morbidity and increased hospital length of stay, both due to the episode of pulmonary edema and potentially due to hypoxemia-related worsening of underlying conditions. This morbidity burden and increased length of stay is associated with increases in hospital costs - a real concern in the current medical economic landscape in the United States. It is reasonable to propose that TACO incidence be considered and monitored as a potentially avoidable medical complication that could be included among other measures of quality of care. This would lend real incentives to reducing the incidence of TACO.

\section{Current limitations in evidence and potential improvements in the prevention, diagnosis, and management of TACO}

This review of literature has revealed several areas where evidence is incomplete or inadequate. In terms of epidemiology, there is inadequate data derived from active surveillance on the incidence of TACO. Further elaboration of a syndromic definition of TACO, perhaps by the National Institute of Health consensus development program (http://consensus. nih.gov/), will help to standardize surveillance and allow comparison between different systems. The use of electronic algorithms to detect TACO, using the electronic medical record, is a new area of research but needs additional work. In the area of prevention, can the detection of patients at high risk for TACO translate to effective preventive measures? And finally, what specific preventive measures can be evaluated by means of interventional trials to compare their efficacy?

As seen in our section on TACO incidence, above, there is a wide discrepancy between data derived from passive surveillance versus that derived from active surveillance. This is not surprising given the general limitations of passive surveillance which is well recognized in the public health literature. ${ }^{13,61}$ We suspect that the true incidence is closer to values derived from studies incorporating active surveillance, and therefore may be as common as $0.5 \%-1.0 \%$ per transfused patient. ${ }^{9,20,21}$ Two other poorly defined areas affect the accurate determination of incidence. First, a better surveillance definition than that given by the CDC Biovigilance Network is needed. ${ }^{6}$ The current definition, based on the presence of one or more signs or symptoms of pulmonary edema, does not require the actual diagnosis of pulmonary edema to fulfill criteria - an important shortcoming since the definition of TACO includes the occurrence of pulmonary edema. Second, experts in critical care medicine may lend their expertise to the elaboration of more precise diagnostic categories for TACO, transfused ALI, and TACO in the setting of preexisting cardiac risk factors. ${ }^{15,33}$ Finally, the incidence of TACO is likely to be a moving target in the setting of substantially declining red blood cell transfusion rates $-15 \%-20 \%$ decrease over the last 5 years - in the United States. ${ }^{76}$ As noted in our section on primary prevention, the most effective way of preventing TACO is to not give the transfusion, and patient blood management strategies currently underway are likely to diminish the overall incidence of TACO, if not necessarily the incidence per transfused patient. A recent meta-analysis suggested that restrictive transfusion strategies decrease the incidence of pulmonary edema (relative risk $=0.48$; CI 0.33-0.72). ${ }^{77}$

This review has revealed reasonable consistency in the definition of TACO risk factors, but further data on their frequency and strength of association with TACO will be important in constructing predictive risk algorithms. This is currently underway as part of the National Heart Lung and Blood Institute (NHLBI) Recipient Epidemiology and Donor Evaluation Study - III (REDS-III) Serious Transfusion Reactions Including Pulmonary Edema (STRIPE) study. ${ }^{78}$ The next step, for which only rudimentary evidence exists at present, is to construct real-time predictive algorithms whereby physicians may be alerted in the electronic medical record to patients whose data shows that they are at increased risk of TACO should they receive a transfusion. ${ }^{13,36,79,80}$ Elaboration of these algorithms and field testing them will be an important research priority.

As reviewed above, there is little data beyond that in the general pulmonary edema literature on the efficacy of specific prevention and treatment interventions for TACO. Potential interventions include: single unit red blood cell orders; slower infusion rates; prophylactic diuretic administration (when, which diuretics, what dosing?); and the use of alternative plasma transfusion such as coagulation factor concentrates instead of fresh frozen plasma to reverse anticoagulation. The search of the clinicaltrials.gov website revealed fewer than five active or completed clinical trials using the keywords pulmonary edema and transfusion. Interventions included the use of shorter versus longer storage duration red blood cell and the use of different target International Normalized Ratio (INR) values to guide correction of coagulation disorders, 
both studied with regard to the outcome of pulmonary edema. Data from observational studies as well as advice from expert advisory committees can help to define the most promising new interventions.

Finally, additional basic and translational research is needed on the development of diagnostic biomarkers for TACO and its differentiation from TRALI and other causes of ALI. Work to date on BNP and NT-proBNP has not yielded a highly predictive marker, but the addition of other markers of cardiac muscle function and/or inflammation is a promising new area of research. ${ }^{32}$

\section{Conclusion}

In concluding this review, it seems appropriate to conceptualize the current research situation and future research needs regarding TACO using the translational research paradigm: a logical progression from basic science to translational to clinical research.

Additional basic research is needed into the pathophysiology of TACO and the development of potential biomarkers for diagnosis. Is the basic pathophysiology entirely hemodynamic, whereby elevated pulmonary venous pressures lead to transudation of fluid into the alveoli or is there component of damage to the alveolar membrane that predisposes or facilitates the condition? The frequent occurrence of TACO in ICU patients with multiple pathologies makes the differentiation of TACO and Possible TRALI difficult but also raises the possibility that ALI pathology may often overlap with TACO. Recent findings regarding biomarkers in TACO also suggest that inflammation may play a role. While not as clear-cut as in TRALI, such research may yield clues to possible preventive strategies.

Additional translational research is needed to develop better approaches to active surveillance for TACO as well as early warning systems to alert clinicians when patients are at increased risk of the condition. Recent research regarding computer algorithms and electronic medical record systems suggests that sensitive algorithms to detect TACO are currently feasible. Unfortunately, these algorithms are still relatively nonspecific and require some level of expert clinicial review to weed out false alarms. Future versions incorporating natural language processing to automatically review free text chest X-ray and clinical reports will contribute to improved specificity. These electronic approaches applied in a systematic fashion to high risk hospitalized populations can form the basis of ongoing active surveillance for TACO incidence. Such an approach is currently being utilized by the REDS-III STRIPE study examining the incidence of and risk factors for TACO. ${ }^{78}$
Additional clinical research is required into the prevention of TACO including studies of alternative transfusion products, patient-specific blood product infusion rates, and utilization of prophylactic diuretics. Results from basic pathophysiology studies may yield insights into inflammation and ALI that may aid in the prevention of TACO - consider that the demonstration of platelet aggregates in TRALI lungs led to a trial of antiplatelet agents in the prevention of TRALI. ${ }^{81}$ Realistically, it is unlikely that entirely new approaches to the treatment of TACO will be developed beyond those discovered for pulmonary edema stemming from other causes. Nevertheless, there may be a role for testing specific novel strategies in the posttransfusion versus generic pulmonary edema setting.

In conclusion, it may be noted that this review of literature found that most publications on TACO were to be found in journals concerned with transfusion medicine as opposed to general clinical medicine. This indicated to us that TACO has received increased recent awareness among the transfusion medicine community which view it as a potentially preventable transfusion reaction. The next challenge will be to raise awareness among clinicians in internal medicine, surgery, and critical care as they are on the front lines in recognizing patients at risk for TACO and potentially implicating preventive strategies.

\section{Funding}

This study was funded by the NHLBI Research Contract HHSN268201100005I (REDS-III Domestic Clinical Site) and an NHLBI Career Award K24-HL-62235 to Dr Murphy.

\section{Disclosure}

The authors report no conflicts of interest in this work.

\section{References}

1. Popovsky MA. Pulmonary consequences of transfusion: TRALI and TACO. Transfus Apher Sci. 2006;34:243-244.

2. Moore SB, Popovsky MA. Posttransfusion pulmonary edema. Transplantation. 1983;35:637-638.

3. Popovsky MA. The Emily Cooley Lecture 2009 To breathe or not to breathe-that is the question. Transfusion. Epub July 12, 2010.

4. Skeate RC, Eastlund T. Distinguishing between transfusion related acute lung injury and transfusion associated circulatory overload. Curr Opin Hematol. 2007;14:682-687.

5. OzierY, Mertes PM. [TRALI and TACO: diagnostic and clinical management of patients]. Transfus Clin Biol. 2009;16:152-158. French.

6. Division of Health Quality Promotion, National Center for Preparedness Detection, and Control of Infectious Diseases, Centers for Disease Control and Prevention. The National Healthcare Safety Network (NHSN) Manual Biovigilance Component Protocol, Hemovigilance Module, 2009. Atlanta, GA: Centres for Disease Control and Prevention. 
7. Popovsky MA. Transfusion-associated circulatory overload: the plot thickens. Transfusion. 2009;49:2-4.

8. Renaudier P, Rebibo D, Waller C, et al. [Pulmonary complications of transfusion (TACO-TRALI)]. Transfus Clin Biol. 2009;16:218-232. French.

9. Rana R, Fernandez-Perez ER, Khan SA, et al. Transfusion-related acute lung injury and pulmonary edema in critically ill patients: a retrospective study. Transfusion. 2006;46:1478-1483.

10. Tobian AA, Sokoll LJ, Tisch DJ, Ness PM, Shan H. N-terminal probrain natriuretic peptide is a useful diagnostic marker for transfusionassociated circulatory overload. Transfusion. 2008;48:1143-1150.

11. Li G, Daniels CE, Kojicic M, et al. The accuracy of natriuretic peptides (brain natriuretic peptide and $\mathrm{N}$-terminal pro-brain natriuretic) in the differentiation between transfusion-related acute lung injury and transfusion-related circulatory overload in the critically ill. Transfusion. 2009;49:13-20.

12. Lieberman L, Maskens C, Cserti-Gazdewich C, et al. A retrospective review of patient factors, transfusion practices, and outcomes in patients with transfusion-associated circulatory overload. Transfus Med Rev 2013;27:206-212.

13. Clifford L, Singh A, Wilson GA, et al. Electronic health record surveillance algorithms facilitate the detection of transfusion-related pulmonary complications. Transfusion. 2013;53:1205-1216.

14. Atwater BD, Thompson VP, Vest RN 3rd, et al. Usefulness of the Duke Sudden Cardiac Death risk score for predicting sudden cardiac death in patients with angiographic ( $>75 \%$ narrowing) coronary artery disease. Am J Cardiol. 2009;104:1624-1630.

15. Ware LB, Matthay MA. Clinical practice. Acute pulmonary edema. N Engl J Med. 2005;353:2788-2796.

16. Clark AL, Cleland JG. Causes and treatment of oedema in patients with heart failure. Nat Rev Cardiol. 2013;10:156-170.

17. Fatalities reported to FDA following blood collection and transfusion: annual sumamry for fiscal year 2012. Available from: http://www.fda gov/biologicsbloodvaccines/safetyavailability/reportaproblem/transfusiondonationfatalities/ucm346639.htm. Accessed October 27, 2014.

18. Hendrickson JE, Hillyer CD. Noninfectious serious hazards of transfusion. Anesth Analg. 2009;108:759-769.

19. Katz EA. Blood transfusion: friend or foe. AACN Adv Crit Care. 2009;20:155-163; quiz 165 .

20. Popovsky MA, Audet AM, Andrzejewski C Jr. Transfusion-associated circulatory overload in orthopedic surgery patients: a multi-institutional study. Immunohematology. 1996;12:87-89.

21. Bierbaum BE, Callaghan JJ, Galante JO, Rubash HE, Tooms RE, Welch RB. An analysis of blood management in patients having a total hip or knee arthroplasty. J Bone Joint Surg. 1999;81:2-10.

22. Kleinman S, Chan P, Robillard P. Risks associated with transfusion of cellular blood components in Canada. Transfus Med Rev. 2003;17 $120-162$.

23. Agence Francaise de Secuirite Sanitaire des Produits de Sante (AFSSAPS). France Annual Hemovigilance Report 2009. Avalable from: http://ansmsantefr/var/ansm_site/storage/original/application/ bb2a6 b52bdb935954bd985d638e0e734pdf. Accessed October 27, 2014.

24. Bolton-Maggs PH. Bullet points from SHOT: key messages and recommendations from the Annual SHOT Report 2013. Transfus Med. 2014;24:197-203.

25. Murphy EL, Kwaan N, Looney MR, et al. Risk factors and outcomes in transfusion-associated circulatory overload. Am J Med. 2013;126:357. e29-e38

26. Li G, Rachmale S, Kojicic M, et al. Incidence and transfusion risk factors for transfusion-associated circulatory overload among medical intensive care unit patients. Transfusion. 2011;51:338-343.

27. Hebert PC, Wells G, Blajchman MA, et al. A multicenter, randomized, controlled clinical trial of transfusion requirements in critical care. Transfusion Requirements in Critical Care Investigators, Canadian Critical Care Trials Group. N Engl J Med. 1999;340:409-417.

28. Villanueva C, Colomo A, Bosch A, et al. Transfusion strategies for acute upper gastrointestinal bleeding. N Engl J Med. 2013;368:11-21.
29. Roubinian N, Toy P, Looney MR, et al. Role of fluid balance and number of blood transfusions in distinguishing TACO and TRALI (Abstract \#S93-040B). Transfusion. 2012;52:50A.

30. Zhou L, Giacherio D, Cooling L, Davenport RD. Use of B-natriuretic peptide as a diagnostic marker in the differential diagnosis of transfusionassociated circulatory overload. Transfusion. 2005;45:1056-1063.

31. Andrzejewski C Jr, Popovsky MA, Stec TC, et al. Hemotherapy bedside biovigilance involving vital sign values and characteristics of patients with suspected transfusion reactions associated with fluid challenges: can some cases of transfusion-associated circulatory overload have proinflammatory aspects? Transfusion. 2012;52:2310-2320.

32. Roubinian N, Looney M, Toy P, et al. Distinguishing pulmonary transfusion reactions using cytokines and cardiopulmonary biomarkers. Preented at: American Thoracic Society Meeting May 2014:A6639.

33. Goldberg AD, Kor DJ. State of the art management of transfusion-related acute lung injury (TRALI). Curr Pharm Des. 2012;18:3273-3284.

34. Looney MR, Roubinian N, Gajic O, et al. Prospective study on the clinical course and outcomes in transfusion-related acute lung injury. Crit Care Med. 2014;42:1676-1687.

35. Vlaar AP, Hofstra JJ, Determann RM, et al. Transfusion-related acute lung injury in cardiac surgery patients is characterized by pulmonary inflammation and coagulopathy: a prospective nested case-control study. Crit Care Med. 2012;40:2813-2820.

36. Toy $\mathrm{P}$, Gajic $\mathrm{O}$, Bacchetti $\mathrm{P}$, et al. Transfusion-related acute lung injury: incidence and risk factors. Blood. 2012;119:1757-1767.

37. Legrand M, Gayat E. Novelties in biomarkers for the management of circulatory failure. Curr Opin Crit Care. 2013;19:410-416.

38. Cheng JM, Akkerhuis KM, Battes LC, et al. Biomarkers of heart failure with normal ejection fraction: a systematic review. Eur J Heart Fail. 2013;15:1350-1362.

39. Gaggin HK, Szymonifka J, Bhardwaj A, et al. Head-to-head comparison of serial soluble ST2, growth differentiation factor-15, and highlysensitive troponin $\mathrm{T}$ measurements in patients with chronic heart failure. JACC Heart Fail. 2014;2:65-72.

40. Popovsky MA. Transfusion and the lung: circulatory overload and acute lung injury. Vox Sang. 2004;87(Suppl 2):62-65.

41. Andrzejewski C Jr, Casey MA, Popovsky MA. How we view and approach transfusion-associated circulatory overload: pathogenesis, diagnosis, management, mitigation, and prevention. Transfusion. 2013;53:3037-3047.

42. Khawaja F, Wagholikor P, Clifford L, Wilson GA, Ognjen G, Kor DJ. Transfusion-associated circulatory overload: hints from the hemodynamics? (Abstract \#S83-040B). Transfusion. 2014;54:51A.

43. Covelli HD, Nessan VJ, Tuttle WK 3rd. Oxygen derived variables in acute respiratory failure. Crit Care Med. 1983;11:646-649.

44. Toy P, Popovsky MA, Abraham E, et al. Transfusion-related acute lung injury: definition and review. Crit Care Med. 2005;33:721-726.

45. Gajic O, Gropper MA, Hubmayr RD. Pulmonary edema after transfusion: how to differentiate transfusion-associated circulatory overload from transfusion-related acute lung injury. Crit Care Med. 2006;34: S109-S113.

46. Wiedemann HP, Wheeler AP, Bernard GR, et al. Comparison of two fluid-management strategies in acute lung injury. $N$ Engl $J$ Med. 2006;354:2564-2575.

47. Gaasch WH. Diagnosis and treatment of heart failure based on left ventricular systolic or diastolic dysfunction. JAMA. 1994;271: $1276-1280$.

48. Zile MR, Brutsaert DL. New concepts in diastolic dysfunction and diastolic heart failure: Part I: diagnosis, prognosis, and measurements of diastolic function. Circulation. 2002;105:1387-1393.

49. Aurigemma GP, Gaasch WH. Clinical practice. Diastolic heart failure. N Engl J Med. 2004;351:1097-1105.

50. Gandhi SK, Powers JC, Nomeir AM, et al. The pathogenesis of acute pulmonary edema associated with hypertension. $N$ Engl J Med. 2001;344:17-22.

51. Brennan JM, Blair JE, Goonewardena S, et al. Reappraisal of the use of inferior vena cava for estimating right atrial pressure. J Am Soc Echocardiogr. 2007;20:857-861. 
52. Dokainish H, Zoghbi WA, Lakkis NM, Quinones MA, Nagueh SF. Comparative accuracy of B-type natriuretic peptide and tissue Doppler echocardiography in the diagnosis of congestive heart failure. Am J Cardiol. 2004;93:1130-1135.

53. Maisel AS, Krishnaswamy P, Nowak RM, et al. Rapid measurement of B-type natriuretic peptide in the emergency diagnosis of heart failure. N Engl J Med. 2002;347:161-167.

54. Lindenfeld J, Albert NM, Boehmer JP, et al. HFSA 2010 Comprehensive Heart Failure Practice Guideline. J Card Fail. 2010;16:e1-e194.

55. Felker GM, Lee KL, Bull DA, et al. Diuretic strategies in patients with acute decompensated heart failure. $N$ Engl $J$ Med. 2011;364:797-805.

56. Aliti GB, Rabelo ER, Clausell N, Rohde LE, Biolo A, Beck-da-Silva L. Aggressive fluid and sodium restriction in acute decompensated heart failure: a randomized clinical trial. JAMA Intern Med. 2013;173: 1058-1064.

57. Masip J, Roque M, Sanchez B, Fernandez R, Subirana M, Exposito JA. Noninvasive ventilation in acute cardiogenic pulmonary edema: systematic review and meta-analysis. JAMA. 2005;294:3124-3130.

58. Gray A, Goodacre S, Newby DE, Masson M, Sampson F, Nicholl J. Noninvasive ventilation in acute cardiogenic pulmonary edema. $N$ Engl J Med. 2008;359:142-151.

59. Weng CL, Zhao YT, Liu QH, et al. Meta-analysis: noninvasive ventilation in acute cardiogenic pulmonary edema. Ann Intern Med. 2010;152: 590-600.

60. Costanzo MR, Saltzberg M, O'Sullivan J, Sobotka P. Early ultrafiltration in patients with decompensated heart failure and diuretic resistance. J Am Coll Cardiol. 2005;46:2047-2051.

61. Clifford L, Jia Q, Yadav H, et al. Characterizing the epidemiology of transfusion associated circulatory overload in surgical transfused patients. Anesthesiology. 2015;122(1):21-28.

62. Escobar GJ, LaGuardia JC, Turk BJ, Ragins A, Kipnis P, Draper D. Early detection of impending physiologic deterioration among patients who are not in intensive care: development of predictive models using data from an automated electronic medical record. J Hosp Med. 2012;7: 388-895.

63. Menis M, Anderson SA, Forshee RA, et al. Transfusion-related acute lung injury and potential risk factors among the inpatient US elderly as recorded in Medicare claims data, during 2007 through 2011. Transfusion. 2014;54:2182-2193.

64. Cooper HA, Rao SV, Greenberg MD, et al. Conservative versus liberal red cell transfusion in acute myocardial infarction (the CRIT Randomized Pilot Study). Am J Cardiol. 2011;108:1108-1111.

65. Popovsky MA, Moore SB. Diagnostic and pathogenetic considerations in transfusion-related acute lung injury. Transfusion. 1985;25:573-577.

66. Sarode R, Milling TJ Jr, Refaai MA, et al. Efficacy and safety of a 4-factor prothrombin complex concentrate in patients on vitamin $\mathrm{K}$ antagonists presenting with major bleeding: a randomized, plasmacontrolled, phase IIIb study. Circulation. 2013;128:1234-1243.

67. Gupta SP, Nand N, Gupta MS, Mohan JC. Haemodynamic changes following blood transfusion in cases of chronic severe anemia: increased safety with simultaneous furosemide administration. Angiology. 1983;34:699-704.
68. Blumberg N, Heal JM, Gettings KF, et al. An association between decreased cardiopulmonary complications (transfusion-related acute lung injury and transfusion-associated circulatory overload) and implementation of universal leukoreduction of blood transfusions. Transfusion. 2010;50:2738-2744.

69. Kor DJ, Kashyap R, Weiskopf RB, et al. Fresh red blood cell transfusion and short-term pulmonary, immunologic, and coagulation status: a randomized clinical trial. Am J Respir Crit Care Med. 2012;185:842-850.

70. Bennett-Guerrero E, Kirby BS, Zhu H, Herman AE, Bandarenko N, McMahon TJ. Randomized study of washing 40- to 42-day-stored red blood cells. Transfusion. 2014;54:2544-2552.

71. Hansen AL, Turner TR, Yi QL, Acker JP. Quality of red blood cells washed using an automated cell processor with and without irradiation. Transfusion. 2014;54:1585-1594.

72. Kor DJ. Point-of-care RBC washing to prevent transfusion-related pulmonary complications. Available from: https://clinicaltrials.gov/ ct2/show/NCT02094118. Accessed February 12, 2014.

73. Li G, Kojicic M, Reriani MK, et al. Long-term survival and quality of life after transfusion-associated pulmonary edema in critically ill medical patients. Chest. 2010;137:783-789.

74. Menis M, Anderson SA, Forshee RA, et al. Transfusion-associated circulatory overload (TACO) and potential risk factors among the inpatient US elderly as recorded in Medicare administrative databases during 2011. Vox Sang. 2014;106:144-152.

75. Magee G, Zbrozek A. Fluid overload is associated with increases in length of stay and hospital costs: pooled analysis of data from more than 600 US hospitals. Clinicoecon Outcomes Res. 2013;5:289-296.

76. Roubinian NH, Escobar GJ, Liu V, et al. Decreased red blood cell use and mortality in hospitalized patients. JAMA Intern Med. 2014;174: 1405-1407.

77. Salpeter SR, Buckley JS, Chatterjee S. Impact of more restrictive blood transfusion strategies on clinical outcomes: a meta-analysis and systematic review. Am J Med. 2014;127:124-131. e3.

78. Kleinman S, Busch MP, Murphy EL, Shan H, Ness P, Glynn SA. The National Heart, Lung, and Blood Institute Recipient Epidemiology and Donor Evaluation Study (REDS-III): a research program striving to improve blood donor and transfusion recipient outcomes. Transfusion. 2014;54:942-955.

79. Narick C, Triulzi DJ, Yazer MH. Transfusion-associated circulatory overload after plasma transfusion. Transfusion. 2012;52:160-165.

80. Bolton-Maggs PH, Cohen H. Serious Hazards of Transfusion (SHOT) haemovigilance and progress is improving transfusion safety. $\mathrm{Br} J$ Haematol. 2013;163:303-314.

81. Ortiz-Munoz G, Mallavia B, Bins A, Headley M, Krummel MF, Looney MR. Aspirin-triggered 15-epi-lipoxin A4 regulates neutrophilplatelet aggregation and attenuates acute lung injury in mice. Blood. 2014;124:2625-2634.

82. Finlay-Morreale HE, Louie C, Toy P. Computer-generated automatic alerts of respiratory distress after blood transfusion. $J$ Am Med Inform Assoc. 2008; 15:383-385.
International Journal of Clinical Transfusion Medicine

\section{Publish your work in this journal}

International Journal of Clinical Transfusion Medicine is an international, peer-reviewed, open access, online journal publishing clinicalexperimental, policy-making and evidence-based practices of all topics pertaining to clinical transfusion medicine. Original research, short reports, reviews, case reports and commentaries are invited.
Dovepress

The manuscript management system is completely online and includes a very quick and fair peer-review system, which is all easy to use. Visit http://www.dovepress.com/testimonials.php to read real quotes from published authors. 